\title{
Max-Planck-Institut für Mathematik Bonn
}

Markov measures and extended zeta functions

by

P. E. T. Jorgensen

A. M. Paolucci

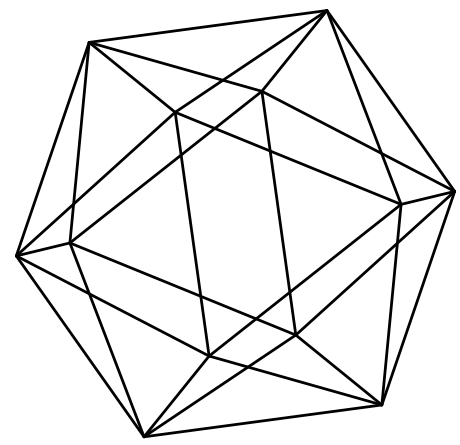





\title{
Markov measures and extended zeta functions
}

\author{
P. E. T. Jorgensen \\ A. M. Paolucci
}

Max-Planck-Institut für Mathematik

Vivatsgasse 7

53111 Bonn

Germany
Department of Mathematics

University of lowa

lowa City, IA 52224

USA 



\title{
Markov measures and extended zeta functions
}

\author{
P.E.T Jorgensen ${ }^{1}$, A.M.Paolucci ${ }^{2}$ \\ ${ }^{1}$ Dept of Mathematics, University of Iowa \\ Iowa City, IA 52224 USA \\ ${ }^{2}$ Max-Planck-Institut für Mathematik \\ Vivatsgasse 7, 53111 Bonn \\ Germany
}

February 3, 2011

\begin{abstract}
In this paper we study a family of representations of the Cuntz algebras $O_{p}$ where $p$ is a prime. These algebras are built on generators and relations. They are $\mathrm{C}^{*}$-algebras and their representations are a part of non-commutative harmonic analysis. Starting with specific generators and relations we pass to an ambient $\mathrm{C}^{*}$-algebra, for example in one of the Cuntz-algebras. Our representations are motivated by the study of frequency bands in signal processing: We construct induced measures attached to those representations which turned out to be related to a class of zeta functions. For a particular case those measures give rise to a class of Markov measures and $q$-Bernoulli polynomials. Our approach is amenable to applications in problems from dynamics and mathematical physics: We introduce a deformation parameter $q$, and an associated family of $q$-relations where the number $q$ is a "quantum-deformation," and also a parameter in a scale of (Riemann-Ruelle) zeta functions. Our representations are used in turn in a derivation of formulas for this $q$-zeta function.
\end{abstract}

Key words: commutator, quantum theory, signal processing, zeta functions, Hilbert space, spectrum.

2000 Mathematics Subject Classification. 47B47, 81P15, 60G35, 33D50, 46C05, 46L52.

\section{Introduction}

In recent papers, there is a renewed interest the use Markov measures; but now in a context of p-adic numbers. An earlier real analysis approach by one of the co-authors and collaborators is motivated by multiresolutions. It makes uses a derivation of Markov measures based on scale-similarity (see e.g., ([16], [25]). 
By contrast, the more recent $p$-adic extensions are motivated in turn by an entirely different set of questions: problems in number theory, in error-correction codes, and in physics; see e.g., [43]. As a result, there is now a need for Markov measures in this rather different context of p-adic analysis; for recent work, see [38], [43]. In section 4 below, we develop Markov measures in the p-adic context, making use of four tools: selfsimilarity, Kolmogorov consistency, a family of representations of the Cuntz algebra $O_{p}$, and a scale of $q$-Bernoulli polynomials (definitions below). In Theorem 12 (section 4) we give a new formula for the $p$-Markov measure on $p$-adic "intervals." We then show that Kolmogorov consistency holds. And using this, in Theorem 15 we compute an associated stochastic process, as well as its joint probability distribution.

Below we offer an overview of the general idea, and we will then turn to the details in the subsequent sections in the paper.

The framework for our results is at the crossroads of the theory of Markov measures, extended zeta functions, and $q$-zeta functions. The three are the subject of recent interesting and interrelated research papers: Markov measures [51], [52], [7], [9] are used in representation theory as well as in statistical mechanics. Extended zeta functions [47] are used in related problems in dealing with quasi-invariant measures. The third, the $q$-zeta functions [38], are also related, and they play a separate role both in number theory and in approximation theory. The use of probability in the study of zeta functions has precursors in the literature; for example it is easy to derive Euler's product formula for the classical zeta function $\zeta$ via an associated probability space, defined directly from $\zeta$ in such a way that all the separate primes define independent events. The extended zeta functions and $q$-zeta functions we consider here will rely on analogous tools; only we will have to resort to non-commutative measure theory: Hence the role played below by algebras of bounded linear operators on Hilbert space; $\mathrm{C}^{*}$-algebras, especially Cuntz algebras and their representations.

The measures we consider here are motivated in part by Jessen and Wintner ([20]); assigning distribution functions (or rather measures) to infinite products and related Zeta functions. However here, a direct approach will not work. The indirect approach which we take is this: Following Riesz and Radon, we view measures as positive linear functionals on a suitably chosen algebra of continuous functions. In our setting, this algebra is naturally embedded as an abelian subagebra of an ambient non-abelian $\mathrm{C}^{*}$-algebra. In our construction, the ambient $\mathrm{C}^{*}$-algebra will be one of the Cuntz algebras. We then proceed to get the our Markov measure as restrictions of states on the Cuntz algebra, the states in turn correspond to representations (of independent interest) via the GNS construction, see sections 3 and 4 below.

The literature. Our paper combines ideas from operator algebras, multiresolutions, Zeta functions, and Markov measures. To make our paper more accessible, we offer below a few pointers to the relevant literature. Readers familiar with one of these areas, but perhaps not the others, may wish to check the following references covering aspects of these areas used below: operator algebras, Cuntz algebras and their representations ([13], [4],[10], [8], [16], [31], $[22],[24],[25],[26],[40])$; multiresolutions and their diverse uses ([1], [12], [3], 
[23], [2], [14] , [27] ) ; Zeta functions ([6], [39], [20], [38], [37], [36]); and Markov measures ([32], [33], [21], [5], [51], [52], [7], [9]). We further use results from harmonic analysis, such as ([19], [29], [15], [17], [18], [49]).

Details. Here our algebraic approach helps: By an iteration scheme applied to the initial generators, we are able in turn to generate sequences of such families; generation by repeated subdivision. We thereby produce such families of projections in a recursive scheme. Depending on the chosen state, we get scalar measures, and these measures in turn will have specific fractal dimensions, or will have $q$-scaling laws. In the first section below, we make precise these correspondences: representations, the specific $\mathrm{C}^{*}$-algebras, our subdivision schemes, projection valued measures, and our specific states on the $\mathrm{C}^{*}$-algebras.

\section{Fundamentals}

In this section we present some material which is needed later. In particular we give some basic definitions from $p$-adic analysis see ([35]) we are going to use in the following sections. A construction of a projection-valued measure as it was done in ([21]) will be recalled. The induced measure attached to that construction is a crucial step throughout the whole paper.

\subsection{The $p$-adic numbers, $p$-adic distributions, Bernoulli distributions}

This material is presented below in the form we need it later, and our presentation further serves to establishes our notation.

\section{$2.2 \quad$ Fourier duality and $p$-adic completions. The $p$-adic integers $\mathbf{Z}_{p}$ and the dual $\mathbf{Z}_{p^{\infty}}$}

Our connections regarding $p$-adic numbers are as follows. Let $p \in \mathbf{Z}_{+}$be a prime $p \in\{2,3,5, \ldots\}$, and let $\mathbf{Z}_{p}=\mathbf{Z} / p \mathbf{Z}$ be the corresponding cyclic groups. We then have two systems of natural mappings:

$$
\mathbf{Z}_{p^{n}} \rightarrow \mathbf{Z}_{p^{n-1}} \text { mult. by } p,
$$

where $\mathbf{Z}_{p^{n}}=\mathbf{Z} / p^{n} \mathbf{Z}$.

The second system consists of the inclusions:

$$
\mathbf{Z}_{p^{n-1}} \rightarrow \mathbf{Z}_{p^{n}}
$$

Combining the two systems we then get:

$$
0 \leftarrow \mathbf{Z}_{p} \leftarrow \mathbf{Z}_{p^{2}} \leftarrow \mathbf{Z}_{p^{3}} \leftarrow \ldots,
$$


and

$$
0 \rightarrow \mathbf{Z}_{p} \rightarrow \mathbf{Z}_{p^{2}} \rightarrow \mathbf{Z}_{p^{3}} \rightarrow \ldots
$$

As a result, for (1), we get the compact projective limit:

$$
\mathbf{Z}_{p^{n}} \leftarrow \mathbf{Z}_{p}
$$

and for (2) the inductive limit

$$
\mathbf{Z}_{p^{n}} \rightarrow \mathbf{Z}_{p^{\infty}}
$$

The first group $\mathbf{Z}_{p}$ is the $p$-adic integers (note compact), and the second $\mathbf{Z}_{p^{\infty}}=$ $\mathbf{Z}\left[\frac{1}{p}\right] / \mathbf{Z}$ is the (discrete) Pointrjagin-dual. We will use $\mathbf{Z}_{p}$ as a compact abelian group with its $p$-adic Haar measure $\mu_{p}$.

Hence $L^{2}$-functions $f($.$) on \mathbf{Z}_{p}$ will allow Fourier series expansions with Fourier coefficients $c($.$) indexed by \mathbf{Z}_{p}$, i.e.,

$$
c(\chi)=\int_{\mathbf{Z}_{(p)}} \bar{\chi}(x) f(x) d \mu_{(p)}(x)
$$

where $\chi \in \mathbf{Z}_{p^{\infty}}$, and

$$
\sum_{\chi \in \mathbf{Z}_{p} \infty}|c(\chi)|^{2}=\int_{\mathbf{Z}_{(p)}}|f(x)|^{2} d \mu_{(p)}(x) .
$$

Let us introduce the $p$-adic norm, i.e. a map: $|.|_{p}: \mathbf{Q} \rightarrow \mathbf{R}_{+}$defined as follows:

$$
|x|_{p}=p^{\operatorname{ord}_{p}(x)}
$$

if $x \neq 0$ and zero otherwise, where $\operatorname{ord}_{p}$ is the $p$-adic order. We denote by $\mathbf{Q}_{p}$ the field of $p$-adic numbers as the completion of the field $\mathbf{Q}$ with respect to the $p$-adic norm. Thus the ring $\mathbf{Z}_{p}$ of $p$-adic integers is a subring of the ring $\mathbf{Q}_{p}$, i.e. $\mathbf{Z}_{p}=\left\{x \in \mathbf{Q}_{p}:|x|_{p} \leq 1\right\}$. The metric given by

$$
\rho_{p}(x, y)=|x-y|_{p} \quad x, y \in \mathbf{Q}_{p}
$$

makes $\mathbf{Q}_{p}$ a complete metric space and since the corresponding metric is nonArchimedean, the corresponding metric $\rho_{p}$ satisfies the strong triangle inequality:

$$
\rho_{p}(x, y) \leq \max \left(\rho_{p}(x, z), \rho_{p}(z, y)\right) \quad x, y, z \in \mathbf{Q}_{p} .
$$

This is called an ultrametric. The $p$-adic norm has a discrete set of values $\left\{p^{r}: r \in \mathbf{Z}\right\} \cup\{0\}$, thus we need to consider balls of radiuses $p^{r}$, where $r \in \mathbf{Z}$. Let us denote by $B_{r}(a)=\left\{x:|x-a|_{p} \leq p^{r}\right\}$ the closed ball of radius $p^{r}$ with the center at a point $a \in \mathbf{Q}_{p}$, the corresponding open ball is denoted by $B_{r}^{-}(a)=$ 
$\left\{x:|x-a|_{p}<p^{r}\right\}$, and by $S_{r}(a)=\left\{x:|x-a|_{p}=p^{r}\right\}$ the sphere of radius $p^{r}$ with the center at a point $a \in \mathbf{Q}_{p}, r \in \mathbf{Z}$. Therefore

$$
\cup_{r} B_{r}(a)=S_{r}(a)=\mathbf{Q}_{p}
$$

and

$$
\cap_{r} B_{r}(a)=\{a\}
$$

Let us note that $B_{0}=\mathbf{Z}_{p}$ is the set of $p$-adic integers, $S_{0}=\mathbf{Z}^{\star}$ is the group of invertible elements (i.e. multiplicative group) in $\mathbf{Z}_{p}$, and $B_{-1}=p \mathbf{Z}_{p}$ is a unique maximal ideal of the ring $\mathbf{Z}_{p}$. A typical interval in $\mathbf{Q}_{p}$ is of the form:

$$
a+p^{n} \mathbf{Z}_{p}=\left\{x \in \mathbf{Q}_{p}:|x-a|_{p} \leq p^{-n}\right\}
$$

The following lemma gives a characterization of a measure on a $p$-adic interval.

Lemma 1 Let $[x]_{q}=\frac{1-q^{x}}{1-q}$. For $d$ a fixed positive integer with $(p, d)=1$ let $X=X_{d}=\lim _{\leftarrow N} \mathbf{Z} / d p^{N} \mathbf{Z}, X_{1}=\mathbf{Z}_{p}, X^{\star}=\bigcup_{(a, p)=1}^{0<a<d p}\left(a+d p^{N} \mathbf{Z}_{p}\right)$, and

$$
a+d p^{N} \mathbf{Z}_{p}=\left\{x \in X / x \cong a\left(\bmod d p^{N}\right)\right\},
$$

$a \in \mathbf{Z}, 0<a<d p^{N}$.

Then for any positive integer $N$,

$$
\mu_{q}\left(a+d p^{N} \mathbf{Z}_{p}\right)=\frac{q^{a}}{\left[d p^{N}\right]}
$$

is a distribution function on $X$.

Proof. (see [36]).

This distribution yields an integral for each non-negative integer $m$ such that

$$
\int_{\mathbf{Z}_{p}}[a]^{m} d \mu_{q}(a)=\lim _{N \rightarrow \infty} \sum_{a=0}^{p^{N}-1}[a]^{m} \frac{q^{a}}{\left[p^{N}\right]} .
$$

Then for $f$ an uniformly differentiable function $\mathcal{U D}\left(\mathbf{Z}_{p}, \mathbf{C}_{p}\right)$ at $a \in \mathbf{Z}_{p}$ we have:

$$
\int_{\mathbf{Z}_{p}} f([x]) d \mu_{q}(x)=\lim _{N \rightarrow \infty} \frac{1}{\left[p^{N}\right]} \sum_{0<x<p^{N}-1} f([x]) q^{a} .
$$

\subsection{Projection-valued measures}

The purpose of this subsection is to show that certain representations of the Cuntz algebras $O_{p}$, where $p$ is a prime, and associated projection-valued measures, offer insight into some classical questions regarding distribution functions. 
Assigning of distribution functions (or rather measures) to infinite products and Zeta functions dates back to Jessen and Wintner ([20]). Here we are concerned with construction of a related family of measures. As it turns out, in some cases, a direct approach will not work.

An indirect approach which we take is this: Recall that by Riesz, a measure is a positive linear functional on some suitably chosen algebra of continuous functions, and often this algebra is naturally embedded as an abelian subalgebra of an ambient non-abelian $\mathrm{C}^{*}$-algebra. We use this fact to advantage below; and in our construction, the ambient $\mathrm{C}^{*}$-algebra will be one of the Cuntz algebras. We will then get the desired measure as a restriction of a state on the Cuntz algebra, the state in turn corresponding to a representation via the GNS representation.

We recall that one denotes by $O_{p}$ the $C^{*}$-algebra generated by $p \in \mathbf{N}$, isometries $S_{0}, \ldots, S_{p-1}$ satisfying

$$
S_{i}^{*} S_{j}=\delta_{i j} \mathbf{1}
$$

and

$$
\sum_{i=0}^{p-1} S_{i} S_{i}^{*}=\mathbf{1}
$$

where $i, j=0, \ldots, p-1$.

We construct certain projection-valued measures by studying subdivisions of compact metric spaces and subdivisions of projections in Hilbert spaces.

Definition 2 Let $(X, d)$ be a compact metric space. For subsets $A \subset X$, define the diameter

$$
|A|:=\sup \{d(x, y) / x, y \in A\} .
$$

$A$ partition of $X$ is a family $\{A(i)\}_{i \in I}$, where $I$ is some index set, such that

$$
\bigcup_{i} A(i)=X,
$$

and

$$
A(i) \bigcap A(j)=\emptyset \text {, if } i \neq j .
$$

Let $p \in \mathbf{Z}_{+}, N \geq 2$ and $\Gamma_{p}:=\{0, \ldots, p-1\}$. Suppose that for each $k \in \mathbf{Z}_{+}$we have a partition into Borel subsets $A_{k}(a)$ indexed by $a \in \Gamma_{p}^{k}=\Gamma_{p} \times \ldots \times \Gamma_{p}$ $k$-times and $|A|=0\left(p^{-c k}\right), c>0$. If every $A_{k+1}(a)$ is contained in some $A_{k}(b)$, we say that $\left\{A_{k}(a)\right\}$ is a $p$-adic system of partitions of $X$.

Definition 3 Let $\mathcal{H}$ be a complex Hilbert space. A partition of projections in $\mathcal{H}$ is a system $\{P(i)\}_{i \in I}$ of projections, i.e.,

$$
P(i)=P(i)^{\star}=P(i)^{2}
$$


such that

$$
P(i) P(j)=0 \text { (orthogonality) }
$$

if $i \neq j$, and

$$
\sum P(i)=1_{\mathcal{H}} \text { (completeness) }
$$

where $1_{\mathcal{H}}$ is the identity operator in $\mathcal{H}$. Suppose that for every $k \in \mathbf{Z}_{+}$we have projections $\{P(a)\}_{a \in \Gamma_{p}^{k}}$ such that every $P(a)_{k+1}$ is contained in some $P(b)_{k}$, i.e. $P(b)_{k} P(a)_{k+1}=P(a)_{k+1}$ then the combined system $\left\{P(a)_{k}\right\}_{k \in \mathbf{Z}_{+}, a \in \Gamma_{p}^{k}}$ is a system of partitions of $1_{\mathcal{H}}$ forming, by $p$-adic subdivisions, an $p$-adic system of projections. We call such system a p-adic system of partitions of $1_{\mathcal{H}}$ into projections.

Definition 4 Denote by $B(X)$ the Borel subsets of the compact metric space $X$. A positive operator-valued function $E$ defined on $B(X)$ is called a $\sigma$ additive measure if, given a sequence $B_{1}, B_{2} \ldots$, in $B(X)$ such that $B_{i} \cap B_{j}=\emptyset, i \neq j$, then

$$
E\left(\bigcup_{i} B_{i}\right)=\sum E\left(B_{i}\right) .
$$

Note that the values $E\left(B_{i}\right)$ are positive operators (or non-negative,

i.e. $\langle\xi, E(B) \xi>\geq 0$ ) so we may take the summation on the right hand side to be convergent in the strong operator topology.

Definition $5 A \sigma$-additive function $E: B(X) \rightarrow$ (positive operators) is called a positive operator measure (PVOM). We say that a PVOM E is an orthogonal projection valued measure if one of the two equivalent conditions (a) and (b) is satisfied

- (a) $E(A \bigcap B)=E(A) E(B)$, for all $A, B \in B(X)$

- (b) $E(A) E(B)=0$ if $A, B \in B(X)$ and $A \bigcap B=\emptyset$.

Note that (b) enables us to state the $\sigma$-additive property for countable disjoint families of Borel sets:

$$
\sum_{i} E\left(A_{i}\right)=E\left(\bigcup_{i} A_{i}\right)
$$

From ([21]) we have the following lemma:

Lemma 6 Let $p \in \mathbf{Z}_{+}, N \geq 2$. Let $(X, d)$ be a compact metric space and let $\mathcal{H}$ be a complex Hilbert space. Let $\left\{A_{k}(a)\right\}_{k \in \mathbf{Z}_{+}, a \in \Gamma_{p}^{k}}$ be a p-adic system of partitions of $X$ and let $\left\{P_{k}(a)\right\}_{k \in \mathbf{Z}_{+}, a \in \Gamma_{p}^{k}}$ be the corresponding $p$-adic system of projections. Then there is a unique normalized orthogonal projection-valued measure $E($.$) defined on the Borel subsets of X$ and taking values in the orthogonal projections in $\mathcal{H}$ such that:

$$
E\left(A_{k}(a)\right)=P_{k}(a)
$$

for every $k \in \mathbf{Z}_{+}, a \in \Gamma_{p}^{k}$. 
Proof. see ([21])

Let the operators $P_{k}(a)$ be defined by

$$
P_{k}(a):=S_{a_{1}} S_{a_{2}} \ldots S_{a_{k}} S_{a_{k}}^{\star} \ldots S_{a_{1}}^{\star}
$$

where $a=\left(a_{1}, a_{2}, \ldots, a_{k}\right) \in \Lambda_{A}^{k}$ are total set in the abelian algebra $C\left(\Lambda_{A}\right)$. For every $k \in \mathbf{Z}_{+}$, the finite sums $\sum_{a \in \Lambda_{A}^{k}} c_{a} \chi_{A_{k}(a)}$ form an algebra of functions $U_{k}$ on $X$ and from the definition of the partition system $\left\{A_{k}(a)\right\}_{k \in \mathbf{Z}_{+}, a \in \Gamma_{p}^{k}}$ it follows that there are natural embeddings $U_{k} \rightarrow U_{k+1}$. Thus the mapping defined for every $k \in \mathbf{Z}_{+}$by

$$
\sum_{a \in \Gamma_{p}^{k}} c_{a} \chi_{A_{k}(a)} \rightarrow \sum_{a \in \Gamma_{p}^{k}} c_{a} P_{k}(a), \quad k \rightarrow \infty,
$$

extends to the algebra $U:=\bigcup_{k \in \mathbf{Z}_{+}} U_{k}$. The algebra $U$ is closed under the $\star$. Denote by $\pi$ the mapping defined above. By the definitions 1 and 2 we have that $\pi\left(f_{1} f_{2}\right)=\pi\left(f_{1}\right) \pi\left(f_{2}\right)$ with $f_{1}, f_{2} \in U$ and $\pi(\bar{f})=\pi(f)^{\star}$, with $f \in U$. We observe that any function $f \in C(X)$ may be uniformly approximated with sequences in $U$ since the sets $A_{k}(a)$ satisfy $|A|=0\left(p^{-c k}\right), c>0$. Then the map $\pi$ by a standard argument from function theory extends from $C(X)$ to all the Baire functions and the extension preserves products and adjoints. Call such extension $\widetilde{\pi}$. Define a projection-valued measure by the following:

$$
E(B):=\widetilde{\pi}\left(\chi_{B}\right)
$$

where $\chi_{B}$ is the indicator function of the set $B$. The measure $\widetilde{\pi}$ is obtained as unique extension from the measure $\pi$ so it follows that $E($.$) satisfies the prop-$ erties of definition 2 and it is countably additive. Also it satisfies $E\left(A_{k}(a)\right)=$ $P_{k}(a)$ for every $k \in \mathbf{Z}_{+}, a \in \Gamma_{p}^{k}$.

Lemma 7 Let $p \in \mathbf{Z}_{+}, N \geq 2$ and let $S_{0}, S_{1}, \ldots, S_{p-1}$ be a representation of $O_{p}$ on a Hilbert space $\mathcal{H}$. For $k \in \mathbf{Z}_{+}$and $a=\left(a_{1}, a_{2}, \ldots, a_{k}\right) \in \Gamma_{p}^{k}$ set

$$
S_{a}:=S_{a_{1}} S_{a_{2}} \ldots S_{a_{k}}
$$

and $P_{k}(a)=S_{a} S_{a}^{\star}$. Then the system $\left\{P_{k}(a)\right\}$ is a system of partitions of $1_{\mathcal{H}}$ into projections.

Proof. The projections $P_{k}(a)$ generate an abelian subalgebra of operators. If two operators $S, T$ are positive on a Hilbert space $\mathcal{H}$ then we say $S \leq T$ if $\langle x, S x\rangle \leq\langle x, T x\rangle$. For a pair of projections $P$ and $Q$ in a Hilbert space $\mathcal{H}$, we have $P \leq Q$ if an only if $\|P h\|^{2} \leq\|Q h\|^{2}$ for all $h \in \mathcal{H}$. Orthogonality holds if an only if $P Q=Q P=0$. Then

$$
\begin{array}{r}
\sum_{i} P_{k+1}(a i)=\sum_{i} S_{i_{1}} \ldots S_{i_{k}} S_{i} S_{i}^{\star} S_{i_{k}}^{\star} \\
\ldots S_{i_{1}}^{\star}=S_{i_{1}} \ldots S_{i_{k}} \sum S_{i} S_{i}^{\star} S_{i_{k}}^{\star} \ldots S_{i_{1}}^{\star}=S_{i_{1}} \ldots S_{i_{k}} S_{i_{k}}^{\star} \ldots S_{i_{1}}^{\star}=P_{k}(a)
\end{array}
$$

using the defining relations of the Cuntz algebra. Thus, $P_{k+1}(a i) \leq P_{k}(a)$, i.e. $P_{k}(a) P_{k+1}(a i) \leq P_{k+1}(a)$. 


\section{Gelfand spaces}

The realization of the representations of the Cuntz algebras $O_{p}$ we have obtained in Section 2 above leads to projection valued measures, and to an important family of abelian $C^{\star}$-algebras. Here we study the Gelfand space of these algebras. They occur naturally as $C^{\star}$-subalgebras of the Cuntz algebra $O_{p}$, here we want to look at them in connection with the system of projections as in Lemma 7.

Let us recall that a finite system of continuous functions $\sigma_{i}: X \rightarrow X$ in a compact metric space $X$ is said to be an iterated function system (IFS) if there is a mapping $\sigma: X \rightarrow X$, onto $X$, such that

$$
\sigma \circ \sigma_{i}=i d_{X}
$$

Given an iterated function system (IFS) such that the system is complete, i.e. $\lim _{k \rightarrow \infty}$ diameter $\left(\sigma_{i_{1}} \circ \ldots \circ \sigma_{i_{k}}(X)\right)=0$ and non-overlapping if for each $k$ the sets

$$
A_{k}\left(i_{1}, \ldots, i_{k}\right):=\sigma_{i_{1}} \circ \ldots \circ \sigma_{i_{k}}(X)
$$

are disjoint, i.e. the sets $A_{k}\left(i_{1}, \ldots, i_{k}\right)$ are mutually disjoint for different multiindices. Then from ([24]) there is a unique projection-valued measure $P$ defined on the Borel subsets of $X$ such that

$$
P\left(A_{k}\left(i_{1}, \ldots, i_{k}\right)\right)=S_{i_{1}} \ldots S_{i_{k}} S_{i_{k}}^{\star} \ldots S_{i_{1}}^{\star},
$$

see (16). Let $C(\Omega)$ the canonical abelian $C^{\star}$-algebra inside $O_{p}$. Specifically, we let $\Omega$ be the Gelfand space of the $C^{\star}$-subalgebra in $O_{p}$ generated by the elements $S_{k_{1}} \ldots S_{k_{m}} S_{k_{m}}^{\star} \ldots S_{k_{1}}^{\star}$ as the multi-indices $k=\left(k_{1}, \ldots, k_{m}\right)$ vary. We use the following:

Lemma 8 Let $\Omega$ be the Gelfand space of the abelian $C^{\star}-$ subalgebra $\mathcal{A}$ in $O_{p}$ generated by the monomials $\left\{S_{k} S_{k}^{\star}: k\right.$ varying over all multi-indices formed from the alphabet $\left.\{1,2, \ldots, p\}\right\}$. Then for every $i \in\{1, \ldots, p\}$, the mapping:

$$
A \in \mathcal{A} \longmapsto S_{i} A S_{i}^{\star} \in \mathcal{A}
$$

induces an endomorphism $\tilde{\sigma}_{i}$ of $\Omega$.

Proof. An easy computation.

Remark If $\left(S_{i}\right)$ are isometries in an Hilbert space $\mathcal{H}$ generating a copy of the Cuntz- $C^{\star}$ - algebra

$$
\sigma(A):=\sum_{i} S_{i} A S_{i}^{\star}
$$

defines an endomorphism $\sigma$ of $B(\mathcal{H})=$ all the bounded operators on $\mathcal{H}$. In fact, modulo an action by unitary matrices

$$
S_{i} \rightarrow \sum_{j} u_{j i} S_{j}
$$


this is a bijection between $\operatorname{End}(B(\mathcal{H}))$ and $\operatorname{Rep}(C u n t z, \mathcal{H})$. The number of generators for the particular Cuntz algebra is called the Powers-index of the endomorphism $\sigma$.

In particular we take $\Omega=\widehat{\mathbf{Z}}$. We should think of the $\widehat{\mathbf{Z}}$ as projective limit of $\mathbf{Z}_{p}, p$ prime. The abelian $C^{\star}$-algebra is generated by words of the form $S_{\{k\}} S_{\{k\}}^{\star}$ where $\{k\}=(1, \ldots, k)$ for some $k \in \mathbf{N}$. Denote by $E$ the projectionvalued measure constructed in $([21])$ onto the abelian $C^{\star}$ algebra $C(\Omega)$ such that

$$
E\left(A_{k}\left(a_{1}, \ldots, a_{k}\right)\right)=S_{\{k\}} S_{\{k\}}^{\star} .
$$

In particular if the induced measure is given by the following formula:

$$
\mu_{f}(.):=<f \mid E(.) f>=\|E(.) f\|^{2}
$$

where $f \in \mathcal{H},\|f\|=1$ and $X=[0,1]$, then $\mu_{f}$ is a probability measure. By choosing the partition system such that the subdivisions are given by the $p$-adic fractions $\frac{\alpha_{1}}{p}+\ldots+\frac{\alpha_{k}}{p^{k}}$ where $\alpha_{i} \in \mathbf{Z}_{p}$.

Then it follows that both the projection valued measure $P($.$) and the indi-$ vidual measures $\mu_{f}()=.\|P(.) f\|^{2}$ are defined on Borel subsets of $[0,1)$.

\section{Cuntz algebras and $p$-adic distributions}

In this section we study commutation relationship between the cases with $S^{\star}$ on the left vs on the right in operator monomials. It will be governed by a deformation parameter. It is of interest in two ways: a quantum deformation and a parameter in a Riemann zeta function. $\operatorname{In}([1])$ we used the $q$-integration in a wavelets construction depending on a parameter $q$ via representations of the Cuntz algebra. Throughout the paper $\mathbf{Q}, \mathbf{C}, \mathbf{Q}_{p}$ and $\mathbf{C}_{p}$ will respectively denote the field of rational numbers, the complex number field, the field of the $p$-adic rational numbers and the completion of the algebraic closure of $\mathbf{Q}_{p}$. When we talk about $q$ we consider the parameter as a complex number and we assume that $|q| \leq 1$ We consider a class of representations of the Cuntz algebra in the space of (complex-valued) functions on the $q p$-adic interval as in Lemma 1 . These representations are unitarily equivalent to the representations constructed by Bratteli and Jorgensen in ([4]). Let $L^{2}\left(\mathbf{Z}_{p}, d \mu_{q}\right)$ be the space of $L^{2}$-integrable functions.

Definition 9 We define the characteristic function of a p-adic interval I by $\chi_{I}(t)$ such that $\chi(t)=0$ if $t \notin 1$ and $\chi(t)=1$ if $t \in I$. .

Remark. Note that in Lemma 9 below, we use a measure on $\mathbf{Z}_{p}$ different from the $p$-adic Haar measure, i.e. a measure $d \mu_{q}$, see ([36]).

Lemma 10 Let $p$ be fixed; $O_{p}$ the Cuntz algebra with $p$ generators $S_{0}, S_{1}, \ldots, S_{p-1}$; let $\mathbf{Z}_{p}$ be the p-adic integers and $d \mu_{q}$ the measure from Lemma 1 ; and let $L^{2}\left(\mathbf{Z}_{p}, d \mu_{q}\right)$ be the corresponding Hilbert space; see section 2.1. For $\left.\xi \in L^{2}\left(\mathbf{Z}_{p}, d \mu_{q}\right)\right)$, 
$x \in \mathbf{Z}_{p}$, and $0 \leq i \leq p-1$.

Set $I_{p, 1}$ be a p-adic interval given by $\left\{\sup (a)+p^{n} \mathbf{Z}_{p}, a \in\{0, \ldots, p-1\}\right\}$ and

$$
\left(S_{i} \xi\right)(x)=\sqrt{q^{p}} \chi_{1}(x-i) \xi\left(\left[\frac{1}{p} x\right]\right) .
$$

Then these operators $S_{i}$ define a representation of $O_{p}$, i.e., the formula for the adjoint operator $S_{i}^{\star}$ is given by (26) below, and we have

$$
\left\{\begin{array}{c}
S_{i}^{\star} S_{j}=\delta_{i, j} I_{L^{2}\left(\mathbf{Z}_{p}, d \mu_{q}\right)}, \quad \text { and } \\
\sum_{i=0, \ldots, p-1} S_{i} S_{i}^{\star}=I_{L^{2}\left(\mathbf{z}_{p}, d \mu_{q}\right)}
\end{array}\right.
$$

Proof. The generators $S_{i}$ in $L^{2}\left(\mathbf{Z}_{p}\right)$ have the following form:

$$
S_{i} \xi(x)=\sqrt{q^{p}} \chi_{1}(x-i) \xi\left(\left[\frac{1}{p} x\right]\right) .
$$

Then, by using the inner product and the generators $S_{i}$ we find the expression for the adjoint operator $S_{i}^{\star}$ as follows:

$$
\begin{aligned}
& \int_{\mathbf{Z}_{p}} S_{i} \xi(x) \eta(x) d \mu_{q}(x)=\int_{\mathbf{z}_{p}} \sqrt{q^{p}} \chi_{1}(x-i) \xi\left(\left[\frac{1}{p}(x-i+i)\right]\right) \eta(x-i+i) d \mu_{q}(x) \\
& \int_{\mathbf{Z}_{p}} \sqrt{q^{p}} \chi_{1}(x-i) \xi\left(\left[\frac{1}{p}(x-i)\right]\right) \eta(i+x-i) d \mu_{q}(x)= \\
& \int_{\mathbf{Z}_{p}} \sqrt{q^{p}} \chi_{1}(x-i) \xi\left(\left[\frac{1}{p}(x-i)\right]\right) \eta\left(i+p \frac{x-i}{p}\right) q^{-p} d \mu_{q}\left(\frac{x-i}{p}\right) \\
& \int_{\mathbf{Z}_{p}} \sqrt{q^{p}} \chi_{1}(x-i) \xi\left(\left[\frac{1}{p}(x-i)\right]\right) \eta\left(i+p \frac{x-i}{p}\right) d \mu_{q}(x)= \\
& \int_{\mathbf{Z}_{p}} \frac{1}{\sqrt{q^{p}}} \xi(x) \eta(i+p x) d \mu_{q}(x)=\int_{\mathbf{Z}_{p}} \xi(x) S_{i}^{\star} \eta(x) d \mu_{q}(x)
\end{aligned}
$$

since $d \mu_{q}(p x)=q^{p} d \mu_{q}(x)$. This follows from the fact that the the measure of $B_{0}$ is $q^{p}$ of the measure of $p B_{0}$, where $B_{0}$ is the ball $\mathbf{Z}_{p}$ union of disjoint balls $B_{-1}(k)=k+p B_{0}, k=0, \ldots, p-1$. Thus

$$
S_{i}^{\star} \xi(x)=\frac{1}{\sqrt{q^{p}}} \xi(i+p x) .
$$

The operators defined by (24) and (26) satisfy the Cuntz algebra relations thus they give a representation of $O_{p}$.

By using the above $p$-adic representation of the Cuntz algebra we can write

$$
S_{i} S_{i}^{\star} \xi(x)=\chi_{1}(x-i) \xi(x) .
$$

Thus, by iterating, we have

$$
<1, S_{I} S_{I}^{\star} 1>=\int_{\mathbf{z}_{p}} \chi_{I_{p, k}}(x-I) d \mu_{q}(x)
$$


where $I_{p, k}=\left\{\sup \left(i_{1}+\ldots+i_{k} p^{k-1}\right)+p^{n} \mathbf{Z}_{p}\right\}$, where $n \geq k$ and $\chi_{I_{p, k}}\left(x-I_{p, k}\right)$ is the corresponding characteristic function.

It is a fact that the $p$-adic representation is equivalent to those permutative representations as in ([4]). Then by considering the iterated function system (IFS) given by $\sigma_{i}(x)=\frac{x+i}{p}$, we have

$$
\sigma_{i_{1}} \circ \ldots \circ \sigma_{i_{k}}[0,1)=\left[\frac{i_{1}}{p}+\ldots+\frac{i_{k}}{p^{k}}, \frac{i_{1}}{p}+\ldots+\frac{i_{k}}{p^{k}}+\frac{1}{p^{k}}\right)
$$

and by $(19)$ we set $A_{k}\left(i_{1}, \ldots, i_{k}\right)=\sigma_{i_{1}} \circ \ldots \circ \sigma_{i_{k}}[0,1)$.

We recall that the induced measure $\mu_{f}$ when $f=1$ is given by :

$$
\begin{array}{r}
\mu_{1}\left(A_{k}\left(i_{1}, \ldots, i_{k}\right)\right) \\
=<1, S_{i_{1}} \ldots S_{i_{k}} S_{i_{k}}^{\star} \ldots S_{i_{1}}^{\star} 1>.
\end{array}
$$

Compare with (23) above.

Thus on $\left(L^{2}\left([0,1], d \mu_{q}\right)\right.$ and for $k=1$ we have

$$
\begin{array}{r}
\mu_{1}\left(A_{1}\left(i_{1}\right)\right)=<1, S_{i_{1}} S_{i_{1}}^{\star} 1>= \\
\int_{\mathbf{Z}_{p}} \chi_{I_{p, 1}}(x-i) d \mu_{q}=\frac{q^{p-1}}{[p]_{q}} .
\end{array}
$$

Observe that by iterating, we have

$$
<1, S_{I} S_{I}^{\star} 1>=\int_{\mathbf{z}_{p}} \chi_{I_{p, k}}(x-I) d \mu_{q}(x)
$$

where $I_{p, k}=\left(i_{1}, \ldots, i_{k}\right)$ and $\chi_{I_{p, k}}\left(x-I_{p, k}\right)$ the characteristic function of the $p$-adic interval $I_{p, k}$.

For any $k>1$ (see Cor 3.5 ([4]):

$$
\mu_{1}\left(A_{k}\left(i_{1}, \ldots, i_{k}\right)\right)=\frac{\left(q^{p-1}\right)^{k}}{[p]_{q}^{k}} .
$$

Thus we have proven the following Lemma:

Proposition 11 Given a representation of $O_{p}$ on $\left(L^{2}\left([0,1), d \mu_{q}\right)\right.$ and let $A_{k}\left(i_{1}, \ldots, i_{k}\right)$ be the above set, then we have

$$
\left.\mu_{1}\left(A_{k}\left(i_{1}, \ldots, i_{k}\right)\right)\right)=\frac{\left(q^{p-1}\right)^{k}}{[p]_{q}^{k}} .
$$

Then the measure for every $k$ of the interval $A_{k}\left(i_{1}, \ldots, i_{k}\right)$ is a product of Bernoulli type measures, e.g. $\mu_{1}\left(A_{1}(i)\right)$. 
Proof. We observe that with the Hilbert space and the inner product $\langle\cdot, \cdot\rangle$ as before, from the above calculation of the induced measure written in terms of the $S_{i}$ and $S_{i}^{\star}$, we have:

$$
\left.\left\|S_{i_{1}} S_{i_{1}}^{\star} 1\right\| \ldots\left\|S_{i_{k}} S_{i_{k}}^{\star} 1\right\|=\mu_{1}\left(A_{k}\left(i_{1}, \ldots, i_{k}\right)\right)\right) .
$$

Thus it follows

$$
\left\|S_{i_{1}} S_{i_{1}}^{\star} 1\right\| \ldots\left\|S_{i_{k}} S_{i_{k}}^{\star} 1\right\|=\left\|S_{i_{k}}^{\star} \ldots S_{i_{1}}^{\star} 1\right\|
$$

which, in a probabilistic setting, it is a condition of independence when the random variable are the $S_{i} S_{i}^{\star}$.

Let us then observe that the measure $\mu_{1}$ constructed in Lemma 7 has analogies with the case of the probability of Bernoulli generated numbers. To see that let us recall then a few known facts about $p$-divisibility and $q$-Riemann zeta functions, see $([49])$. For $p$ a prime an $s$-tuple $\left(n_{1}, \ldots, n_{s}\right)$ of natural numbers is said to be $p$-divisible if $p$ divides each $n_{j}$. Using this notion of $p$-divisibility for the case of Bernoulli trials a $q$ - Riemann zeta function arises. For $m \in \mathbf{Z}^{+}$, let $\overline{1}, \ldots, \bar{m}$ be the set of congruences classes modulo $m$. Since $[m]_{q}=\frac{1-q^{m}}{1-q}$ it follows for $0 \leq q<1$ that the probability of a Bernoulli generated number $n$ belonging to the class $\bar{r}$ is

$P_{q}(n \in \bar{r}):=\sum_{k} q^{r-1+k m}(1-q)=(1-q) q^{r-1} \sum_{k}\left(q^{m}\right)^{k}=\frac{(1-q) q^{r-1}}{1-q^{m}}=\frac{q^{r-1}}{[m]}$ since $\frac{q^{r-1}}{[m]}$ is continuous from the left at $q=1$ then $P_{q}$ may be continuously extended on congruences classes to $q=1$ by defining $P_{1}(n \in \bar{r})=\frac{1}{m}$ for $r=1, \ldots, m$.

Hence the extended $P_{q}$ is a $q$-analog of the equiprobable measure since all numbers $\mathbf{Z}^{+}$are equally likely to come up.

For $n \in \mathbf{Z}^{+}$, let $n=p_{1}^{\alpha_{1}} p_{2}^{\alpha_{2}} \ldots p_{k}^{\alpha_{k}}$, where $p_{1}, \ldots, p_{k}$ are distinct primes and $\alpha_{1}, \ldots, \alpha_{k}$ are positive integers.

Thus the $q$ canonical factorization of $n$ is defined to be $\{n\}=\left[p_{1}\right]^{\alpha_{1}}\left[p_{2}\right]^{\alpha_{2}} \ldots\left[p_{k}\right]^{\alpha_{k}}$. Set $\nu(n)=\alpha_{1}\left(p_{1}-1\right)+\alpha_{2}\left(p_{2}-1\right)+\ldots+\alpha_{k}\left(p_{k}-1\right)$ with the convention that $\nu(1)=1$. For $s>1$ and $0 \leq q<1$ a $q$ analog of the Riemann zeta function is defined by

$$
\zeta_{q}(s)=\sum_{n} \frac{q^{s \nu(n)}}{\{n\}^{s}} .
$$

Then for $s>1$ and $0 \leq q<1$ the zeta function $\zeta_{q}(s)$ is convergent and it has the form

$$
\zeta_{q}(s)=\prod_{p}\left(\frac{1-q^{s(p-1)}}{[p]^{s}}\right)^{-1}
$$


where the product is over all primes.

If we consider repeating the Bernoulli scheme $s$ times for every $p$ then this will result in a sequence of $s$ - tuples each of the form $\left(n_{1}, n_{2}, \ldots, n_{s}\right)_{p}$. Since the components of such an $s$-tuple are determined independently it is known that the probability of $n$ not being divisible is

$$
1-\prod_{j} P_{q}\left(n_{j} \in \bar{p}\right)=1-\prod \frac{q^{p-1}}{[p]}=1-\frac{q^{s(p-1)}}{[p]^{s}} .
$$

Provided that $s>1$ the probability of $\left\{n_{1}, \ldots, n_{s}\right\}$ not being $p$ divisible for every prime $p$ is given by $\Pi\left(1-\frac{q^{s(p-1)}}{[p]^{s}}\right)$ and this infinite product converges to $\frac{1}{\zeta_{q}^{(s)}}$. Observe that by the above construction the induced measure for $k=1$ and $f=1$ where $A_{1}(i)$ is the $p$-adic interval is :

$$
\mu_{1}\left(A_{1}\right):=<1, S_{i_{1}} S_{i_{1}}^{\star} 1>=\frac{q^{p-1}}{[p]_{q}}
$$

it agrees with the value of the probability of a Bernoulli generated number. Then it is a standard fact that it is related to the $q$ - zeta function introduced above. Let us denote the measure constructed above by $\mu_{1}$ to denote the dependence on the choice of the prime $p$.

For a prime $p$, let $\left(n_{1}, n_{2}, \ldots, n_{s}\right)$ be an $s$-tuple of natural numbers where $p$ divides each $n_{j}$ and $A_{1}\left(n_{i}\right)$ and $A_{s}\left(n_{1}, n_{2}, \ldots, n_{s}\right)$ are as above.

Then, for every $n_{i}$, the measure is:

$$
\mu_{1}\left(A_{1}\left(n_{i}\right)\right)=\frac{1}{[p]_{q}} q^{p-1}
$$

for each $i=1, \ldots, s$.

Thus,

$$
\begin{array}{r}
1-\prod_{i=1, \ldots, s} \mu_{1}\left(A_{1}\left(n_{i}\right)\right)=1-\prod_{i=1, \ldots, s} \frac{1}{[p]_{q}} q^{p-1} \\
=1-\frac{1}{[p]_{q}^{s}} q^{s(p-1)}
\end{array}
$$

for every $p$ and $\mu_{1}\left(A_{s}\left(n_{1}, n_{2}, \ldots, n_{s}\right)\right)=\frac{1}{[p]_{q}^{s}} q^{s(p-1)}$ from Proposition 11 . We apply the construction of the infinite product measure see ([23]) since the Kolmogorov compatibility condition is satisfied. Then it follows that $\mu_{1}$ is a probability measure.

Denote by $\mu_{1}\left(A_{s}\left(n_{1}, n_{2}, \ldots, n_{s}\right)\right)=\mu_{1}(s)$ since it depends on the variable $s$ appearing in the $p$-adic interval $A_{s}\left(n_{1}, n_{2}, \ldots, n_{s}\right)$.

Thus we have: 
Theorem 12 For every prime $p$ there is a probability measure $\mu_{1}$ induced from operator projection-valued measure associated to the Cuntz algebra $O_{p}$ such that:

$$
\prod_{p}\left(1-\mu_{1}\left(A_{s}\left(n_{1}, \ldots, n_{s}\right)\right)\right)
$$

converges to $\frac{1}{\zeta_{q}(s)}$ where $\zeta_{q}(s)$ is a q-Riemann zeta function.

Proof. For a prime $p$ an $r$-tuple $\left(n_{1}, n_{2}, \ldots, n_{r}\right)$ of natural numbers is said to be $p$-divisible if $p$ divides each $n_{i}$. Thus the notion of $p$-divisibility applied in the context of Bernoulli trials gives rise to a probabilistic application of the $q$ - Riemann zeta function, see ([49]). The measure constructed above from an operator theoretic approach via Cuntz algebras is a probability measure. It is a $p$-divisible measure since $p$ divides each $n_{i}$ by construction of the maps $\sigma_{i}$. Let

$$
\prod_{i} \mu_{1}
$$

be the product measure constructed from the family of measures $\mu_{1}$ constructed above.

Therefore by standard results there exists an independent product measures space such that the product measure of $\mu_{1}$ is a probability measure. Since from Theorem 2([49]) we get that the infinite product

$$
\prod_{p}\left(1-\frac{q^{r(p-1)}}{[p]^{r}}\right)
$$

converges to $\frac{1}{\zeta_{q}(s)}$. This proves the statement of the theorem.

In the language of probability we can see this formula as the statement that the $\zeta$-probability on the events $\{p \mathbf{N}: p \in$ all primes $2,3,5, \ldots\}$ are independent.

\section{$5 \quad$ Markov measures}

In this section we look into the construction of a Markov measure via the induced measures introduced in Section 2. A Markov measure is built out of the induced measure $\mu_{f}$ of the previous section for a particular choice of the functions $f$. This turns out to be a $p$-adic distribution giving rise to Bernoulli polynomials and having a Markovian behaviour. When we choose the function $f=1, m=0$ we get the results of the previous section. We want to point out that in this construction we use the definition of the Bernoulli polynomials as in ([36]) and the measure in the integral definition of the Bernoulli polynomials is the $q$ measure $\mu_{q}$ which we have defined on Section 2 .

Set $I_{p, i}$ to be the $p$-adic interval $\left(i+p \mathbf{Z}_{p}\right)$ for every $p$ prime. From Lemma 1 we have

$$
\mu_{q}\left(I_{p, i}\right)=\frac{q^{i}}{[p]_{q}} .
$$


Iterating (32) we get the following:

$$
\begin{array}{r}
\mu_{q}\left(i_{1}+i_{2}+\ldots+i_{k}+p^{k} \mathbf{Z}_{p}\right) \\
=\frac{q^{i_{1}+i_{2}+\ldots+i_{k}}}{\left[p^{k}\right]_{q}} .
\end{array}
$$

Let us recall that any open-closed subset in $\mathbf{Z}^{\star}$ is a disjoint union of some family of finite intervals $I_{a, n}=a+p^{n} \mathbf{Z}_{p}$, as defined in the previous section. Let us denote by $I_{(K)}$ the $p$-adic interval where $K=\left\{i_{1} \ldots i_{k}\right\}$. Consider the functions

$$
f_{m, q}\left(I_{i_{1}, \ldots, i_{k}, n}\right)=\left[p^{n}\right]^{m-1} B_{m}\left(\frac{i_{1}+\ldots+i_{k}}{p^{n}}: q^{p^{n}}\right)
$$

where $B_{m}(x: q)$ are the $p$-adic $q$-Bernoulli polynomials given in the integral representation by

$$
B_{m}(x: q)=\int_{\mathbf{z}_{p}}[x+t]^{m} q^{-t} d \mu_{q}(t) .
$$

In particular when $m=1$, we have

$$
B_{1}(x: q)=\int_{\mathbf{z}_{p}}[x+t] q^{-t} d \mu_{q}(t) .
$$

Lemma 13 Let the functions $\nu_{m, q}$ be defined on a p-adic interval $I_{a, n}=\left\{a+p^{n} \mathbf{Z}\right\}$ by

$$
\nu_{m, q}\left(I_{a, n}\right)=\left[p^{n}\right]^{m-1} B_{m}\left(\frac{a}{p^{n}}: q^{p^{n}}\right) .
$$

Then $f_{m, q}$ is a $\mathbf{Q}_{p}(q)$ - measure on $\mathbf{Z}^{\star}$.

Proof.

See $([36])$.

Let $I_{m, i_{j}, f}=\left\{f_{m}\left(\omega, i_{1}\right), \omega \in \mathbf{Z}_{p}\right\}$, where the functions $f$ are taken as $f_{m}\left(\omega, i_{j}\right)=$ $\left[\omega+i_{j}\right]^{m}$. We consider a sequence of random variables $X_{i_{j}}$ such that

$$
X_{i_{1}}(\omega) \in I_{m, i_{1}, f}, \ldots, X_{i_{k}}(\omega) \in I_{m, i_{k}, f} .
$$

The next corollary shows that the distribution of the process $\left\{X_{i_{j}}\right\}$ is given by Bernoulli polynomials with respect to the induced measure of Section 4 .

Corollary 14 Let $I_{m, i_{1}, f}$ be defined as

$$
I_{m, i_{1}, f}=\left\{f_{m}\left(\omega, i_{1}\right), \omega \in \mathbf{Z}_{p}\right\} .
$$

Then there exists a measure $P$ such that:

$$
P\left(X_{i_{1}}(\omega) \in I_{1, i_{1}, f}\right)=B_{1}\left(\frac{i_{1}}{p^{n}}: q^{p^{n}}\right) .
$$


Proof. We take the induced measure $\mu_{f}$ constructed in Section 4. Let the function $f_{m}$ be given by $f_{1}: f\left(\omega, i_{1}\right)=\left[\omega+i_{1}\right]$ when $m=1$

Then

$$
\begin{array}{r}
P\left(X_{i_{1}}(\omega) \in I_{1, i_{1}}\right)=\left|<f_{1}, S_{\omega} S_{\omega}^{\star} f_{1}>\right| \\
\quad=\int_{\mathbf{Z}_{p}} \chi_{I_{1, i_{1}}}\left(t-i_{1}\right)\left[\left(i_{1}+t\right)\right] q^{-t} d \mu_{q}(t)
\end{array}
$$

where we denote by $[x]_{q}=\frac{1-q^{x}}{1-q}$.

Using the measure $\mathbf{P}$ as in the previous corollary, we define the joint probability to be:

$$
\begin{array}{r}
\mathbf{P}\left(X_{i_{1}}(\omega) \in I_{\left(1, i_{1}, f_{1}\right)}, \ldots, X_{i_{k}}(\omega) \in I_{\left(1, i_{k}, f_{1}\right)}\right)= \\
\mu_{f_{1}}\left(A_{1}\left(i_{1}\right)\right) \ldots \mu_{f_{1}}\left(A_{1}\left(i_{1}, \ldots, i_{k}\right)\right)=B_{1}\left(\left[i_{1}: q\right]\right) \ldots B_{1}\left(\left[i_{1}+\ldots+i_{k}: q\right]\right) .
\end{array}
$$

Thus we get the following:

Theorem 15 Given the measure as in Corollary 14 then we have

$$
\begin{array}{r}
\mathbf{P}\left(X_{i_{1}}(\omega) \in I_{\left(1, i_{1}, f_{1}\right)} \mid X_{i_{2}}(\omega) \in I_{\left(1, i_{2}, f_{1}\right)}, \ldots, X_{i_{k}}(\omega) \in I_{\left(1, i_{k}, f_{1}\right)}\right) \\
=\mathbf{P}\left(X_{i_{1}}(\omega) \in I_{\left(1, i_{1}, f_{1}\right.}\right) .
\end{array}
$$

Therefore the measure $\mathbf{P}$ is a Markov measure.

Proof. This can be seen by the following:

$$
\begin{array}{r}
\mathbf{P}\left(X_{i_{1}}(\omega) \in I_{\left(1, i_{1}, f_{1}\right)} \mid X_{i_{2}}(\omega) \in I_{\left(1, i_{2}, f_{1}\right)}, \ldots, X_{i_{k}}(\omega) \in I_{\left(1, i_{k}, f_{1}\right)}\right) \\
B_{1}\left(\left[i_{1}: q\right]\right)=\mathbf{P}\left(X_{i_{1}}(\omega) \in I_{1, i_{1}, f_{1}}\right) .
\end{array}
$$

Thus the statement holds.

Acknowledgments P.J. was supported in part by a grant from the National Science Foundation (USA). A.M.P. would like to thank S. Troiani for helpful conversations and the Max-Planck Institut für Mathematik in Bonn for support and excellent working conditions.

\section{References}

[1] S. Albeverio, P. E. T. Jorgensen and A. M. Paolucci, 'Multiresolution wavelets analysis of integer scale Bessel functions', J. Math. Phys. 48, (2007), 073516.

[2] L.W. Baggett, N. S. Larsen, K.D. Merrill, J.A. Packer and I. Raeburn, 'Generalized Multiresolution analyses with give multiplicity functions', to appear Journal of Fourier Analysis and Applications. 
[3] O. Bratteli and P.E.T. Jorgensen, 'Isometries, shifts, Cuntz algebras and multiresolution wavelet analysis of scale $p$,' Integral Equations Operator Theory 28, (1997), 382-443.

[4] O. Bratteli and P.E.T. Jorgensen, 'Iterated function systems and permutation representations of the Cuntz algebra,'Mem. Amer. Math. Soc. 139, no. 663, (1999).

[5] Bratteli O., Jorgensen P.E.T., 'Wavelets through a looking glass', Applied and Numerical Harmonic Analysis, Birkhäuser Boston Inc. Boston, MA, xxii+398, ISBN 0-8176-4280-3, (2002).

[6] L. Carlitz, 'q- Bernoulli numbers and polynomials', Duke Math. J. 15, (1948), 987-1000.

[7] J.-R. Chazottes, E. Ugalde, Projection of Markov measures may be Gibbsian J. Statist. Phys 111, No.5-6, (2003), 1245-1272.

[8] Cho I., Jorgensen P., ' $C^{\star}$-algebras generated by partial isometries', $J$. Appl. Math. Comput., 26, no.1-2, ISSN 5865, (2008), 1-48.

[9] H. Crauel, Markov measures for random dynamical systems, Stochastics and Stochastics Reports 37, No. 3, (1991), 153-173.

[10] J. Cuntz, 'Simple $C^{*}$-algebras generated by isometries', Comm. Math. Phys. 56, (1977), 173-185.

[11] J. Cuntz, 'A Class of $C^{\star}-$ algebras and Topological Markov Chains', Inventiones math 56, (1980), 251-268.

[12] I. Daubechies, 'Ten Lectures on Wavelets', CBMS-NSF Regional Conf. Ser. in Appl. Math. (Society for Industrial and Applied Mathematics) 61, Philadelphia, (1992).

[13] K.R.Davidson, D.R. Pitts, 'Invariant subspaces and hyper-reflexivity for free semigroups algebras', Proc. London Math. Soc., 78, (1999), 401-430.

[14] Dutkay D. E. , Jorgensen P. E. T., 'Wavelets on fractals', Rev. Mat. Iberoam. 22, no. 1, ISSN 0213-2230, (2006), 131-180.

[15] Dutkay D. E., Jorgensen P. E.T., 'Harmonic analysis and dynamics for affine iterated function systems', Houston J. Math. 33, no. 3, (2007), 877-905.

[16] Dutkay D. E. Jorgensen P. E.T., 'Martingales, endomorphisms, and covariant systems of operators in Hilbert space', J. Operator Theory 58, no. 2, (2007), 269-310.

[17] Dutkay, D. E., Jorgensen P. E. T., 'A duality approach to representations of Baumslag-Solitar group, Group representations, ergodic theory, and mathematical physics: a tribute to George W. Mackey', Contemp. Math. 449, Amer. Math. Soc., Providence, RI, (2008), 99-127. 
[18] Dutkay D. E., Jorgensen P. E. T., 'Fourier series on fractals: a parallel with wavelet theory, Radon transforms, geometry, and wavelets', Contemp. Math. 464, Amer. Math. Soc., Providence, RI, (2008), 75-101.

[19] G. Gasper and M. Rahman, ' Basic Hypergeometric Series' 35 Encyclopedia of Mathematics and Its Applications (Cambridge Univ. Press), Cambridge, (1990).

[20] B. Jessen, A. Wintner, 'Distribution Functions and the Riemann Zeta Function', Transactions of the American Math. Soc. 38, (1935), 48-88.

[21] P.E.T Jorgensen, 'Measures in wavelet decompositions', Advances in Applied Mathematics 34, (2005), 561-590.

[22] Jorgensen P. E. T., Analysis and probability: wavelets, signals, fractals, Graduate Texts in Mathematics 234, Springer,New York,xlviii+276, (2006).

[23] P.E.T. Jorgensen, 'Analysis and Probability, Wavelets, Signals, Fractals', Graduate Texts in Mathematics 234, Springer, (2006).

[24] Jorgensen P. E. T., 'Use of operator algebras in the analysis of measures from wavelets and iterated function systems', Contemp. Math. 414, Amer. Math. Soc., Providence, RI, (2006), 13-26.

[25] Jorgensen P. E. T., 'Certain representations of the Cuntz relations, and a question on wavelets decompositions', Contemp. Math. 414, Amer. Math. Soc., Providence, RI, (2006), 165-188.

[26] Jorgensen, P. E. T., 'Unitary matrix functions, wavelet algorithms, and structural properties of wavelets', Lect. Notes Ser. Inst. Math. Sci. Natl. Univ. Singap. 10, World Sci. Publ., Hackensack, NJ, (2007), 107-166.

[27] Jorgensen, P. E. T., 'Frame analysis and approximation in reproducing kernel Hilbert spaces', Contemp. Math. 451, Amer. Math. Soc., Providence, RI, (2008), 151-169.

[28] Jorgensen, P. E. T. , Kornelson K. and Shuman K., 'Orthogonal exponentials for Bernoulli iterated function systems', Appl. Numer. Harmon. Anal., Birkhäuser Boston, Boston, MA, (2008), 217-237.

[29] P. E. T. Jorgensen, K. D. , Merrill and J. A. Packer, (Editors), 'Representations, wavelets, and frames', Applied and Numerical Harmonic Analysis, Birkhäuser Boston Inc.,Boston, MA, xl+322, (2008).

[30] P. E. T. Jorgensen, A. M. Paolucci, 'Wavelets in mathematical physics: q-oscillators', J. Phys. A: Math. Gen. 36, (2003), 6483-6494. 
[31] Jörgensen P. E. T., Proskurin D. P., Samoı̌lenko, Yuriǔ S., 'Generalized canonical commutation relations: representations and stability of universal enveloping $C^{*}$-algebra', Symmetry in nonlinear mathematical physics, Pr. Inst. Mat. Nats. Akad. Nauk Ukr. Mat. Zastos. 2, Natsīonal. Akad. Nauk Ukraïni Inst. Mat., Kiev, (2002), 456-460.

[32] P.E.T. Jorgensen, L.M. Schmitt, and R.F. Werner, ' $q$-canonical commutation relations and stability of the Cuntz algebra', Pacific J. Math. 165, (1994), 131-151.

[33] P.E.T. Jorgensen and R.F. Werner, 'Coherent states of the $q$-canonical commutation relations', Comm. Math. Phys. 164, (1994), 455-471.

[34] K. Kawamura, 'The Perron-Frobenius operators, invariant measures and representations of the Cuntz-Krieger algebra', J. Math. Phys. 46, (2005), 083514.

[35] N. Koblitz,'p-adic Numbers, p-adic Analysis and Zeta -Functions', Springer-Verlag 58, (1977).

[36] T. Kim, 'On p-adic q-Bernoulli numbers', J. Korean Math. Soc. 37, No. 1, (2000), 21-30.

[37] Taekyun Kim, Cheon Seoung Ryoo, 'p-adic $q$-integrals and basic $q$-zeta function', Journal of Concrete and Applicable Mathematics 6, No. 4, (2008), 349-356.

[38] T. Kim, 'Note on the Euler q-zeta functions', Journal of Number Theory 129, No. 7, (2009)1798-1804.

[39] N. Koblitz, On Carlitz's q Bernouilli numbers, J. Number Theory 14, (1982), 332-339.

[40] E.C. Lance, A.M.Paolucci, 'Conjugation in braided $C^{\star}$-categories and orthogonal quantum groups', J.Math. Phys., 41, 4, (2000), 2383-2394.

[41] M.L.Lapidus and M. van Frankenhuijsen, ' Fractal Geometry, Complex Dimensions and Zeta Functions', Springer Monographs in Mathematics, (2006).

[42] F.Luef, Y. I. Manin, 'Quantum theta functions and Gabor frames for modulation spaces', arXiv:0809.2716v2 [math.QA].

[43] Y.I.Manin, M. Marcolli, 'Error-correcting codes and phase transitions' to appear in Mathematics in Computer Science.

[44] M. Marcolli, 'Arithmetic Noncommutative Geometry', AMS Lecture Series $\mathbf{3 6}$, (2005). 
[45] M. Marcolli, 'Modular curves, $C^{\star}$-algebras, and chaotic cosmology', Frontiers in Number Theory, Physics and Geometry, II, Springer, (2006), 361-372.

[46] M. Marcolli, A.M. Paolucci, 'Cuntz-Krieger algebras and wavelets on fractals', Complex Analysis and Operator Theory, DOI 10.1007/s11785009-0044-y.

[47] G. Pantsulaia, 'Invariant and quasiinvariant measures in infinitedimensional topological vector spaces', Nova Science Publishers Inc., New York, (2007).

[48] Raeburn I., 'Graph Algebras', CBMS, (2005).

[49] Rawlings D., 'Bernoulli trials and number theory', The American Mathematical Monthly 101, 10, (1994), 948-952.

[50] M.Ram Murty, 'Introduction to $p$-adic Analytic Number theory', Studies in Advanced mathematics, AMS/IP 27, (2002).

[51] A.M. Vershik, N.V. Tsilevich, 'Markov measures on Young tableaux and induced representations of an infinite symmetric group, Teor. Veroyatn. Primen., Rossiǔskaya Akademiya Nauk. Teoriya Veroyatnosteú i ee Primeneniya 51, No.1, (2006) 47-63.

[52] I. Werner, 'The generalized Markov measure as an equilibrium state', Nonlinearity 18, No. 5, (2005), 2261-2274. 